\title{
Ventilación espontánea en decubito prono en pacientes con infección por SARS Cov-2 sin ventilación mecánica invasiva
}

\section{Spontaneous ventilation in the prone decube in patients with SARS Cov-2 infection without invasive mechanical ventilation}

Claudia Lizeth Uriol-Mantilla,a ${ }^{1, a}$ Gustavo Adolfo Vasquez-Tirado, ${ }^{1, \mathrm{~b}}$

\begin{abstract}
RESUMEN
Introducción. La infección por coronavirus tipo 2 del síndrome respiratorio agudo severo (SARS-CoV-2) causante de la enfermedad por coronavirus 2019 (COVID-19), ha transformado de manera significativa la forma de cómo se practica la medicina hospitalaria en estos últimos 4 meses. Actualmente, existe una gran cantidad de pacientes que ingresan por emergencia a los establecimientos de salud presentando insuficiencia respiratoria que rápidamente evoluciona al estado crítico de la enfermedad, requiriendo en algunos casos el ingreso a la unidad de cuidados intensivos (UCl) y el aporte de ventilación mecánica invasiva (VMI). Se ha llevado a cabo distintas estrategias y métodos para el manejo de pacientes con infección por SARS CoV 2, una de ellas y con mayor relevancia es la posición en decúbito prono (DP), utilizada para mejorar los niveles de oxigenación en pacientes con síndrome de dificultad respiratoria aguda (SDRA). La posición en DP mejora los niveles de oxigenación, disminuyendo el gradiente gravitacional de la presión pleural, mejorando la perfusión y ventilación del parénquima pulmonar, aumentando la capacidad residual funcional y optimizando la relación ventilación/perfusión (V/Q) de los pulmones lesionados. Diversos estudios demuestran el gran beneficio de la posición en DP en pacientes con infección por SARS CoV 2; por lo que actualmente está siendo utilizada como un tratamiento no invasivo para prevenir el deterioro de la función ventilatoria con posterior intubación e ingreso a $\mathrm{UCl}$.
\end{abstract}

Palabras Clave: posición prona, COVID-19, no intubación endotraqueal Fuente: DeCS-BIREME).

\section{ABSTRACT}

Introduction. The severe acute respiratory syndrome type 2 coronavirus infection (SARS-CoV-2) that causes coronavirus disease 2019 (COVID-19) has significantly transformed the way hospital medicine is practiced in these last 4 months. Currently, there are a large number of patients who are admitted to health facilities by emergency presenting respiratory failure that rapidly evolves to the critical state of the disease, requiring in some cases admission to the intensive care unit (ICU) and the provision of ventilation invasive mechanics (IMV). Different strategies and methods have been carried out for the management of patients

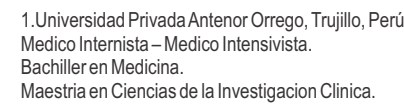

with SARS CoV 2 infection, one of them and with greater relevance is the prone position (PD), used to improve oxygenation levels in patients with syndrome of acute respiratory distress (ARDS). The PD position improves oxygenation levels, decreasing the gravitational gradient of pleural pressure, improving perfusion and ventilation of the lung parenchyma, increasing functional residual capacity and optimizing the ventilation / perfusion ( $\mathrm{V} / \mathrm{Q}$ ) ratio of the injured lungs. Several studies show the great benefit of the PD position in patients with SARS CoV 2 infection; Therefore, it is currently being used as a non-invasive treatment to prevent deterioration of ventilatory function with subsequent intubation and admission to the ICU.

Keywords: prone position, COVID-19, no endotracheal intubation (Source: DeCS-BIREME). 
La pandemia de la enfermedad por coronavirus 2019 (COVID-19) ha transformado de manera significativa la forma de cómo se practica la medicina hospitalaria en estos últimos 4 meses, convirtiéndose en una crisis mundial de la salud donde los médicos hoy en día se enfrentan a un desafío sin precedentes al tratar con pacientes COVID-19. ${ }^{(1)}$ Algunos pacientes con COVID-19 desarrollan síndrome de dificultad respiratoria aguda (SDRA), llevando al paciente al estado crítico de la enfermedad y requiriendo el ingreso a la unidad de cuidados intensivos ( $\mathrm{UCl}$ ) y el aporte de ventilación mecánica invasiva $(\mathrm{VMI}) .^{(2)}$ Debido a la crisis que estamos atravesando, los recursos médicos se han agotado rápidamente, lo que impulsa a los médicos a realizar estrategias de apoyo terapéutico y de oxigenación para evitar el deterioro ventilatorio y la muerte del paciente. ${ }^{(3)}$ Dentro de ello tenemos la posición en decúbito prono (DP), estrategia utilizada para mejorar la oxigenación arterial en pacientes con SDRA. Se ha demostrado que la posición en DP mejora la relación ventilación/perfusión $(\mathrm{V} / \mathrm{Q})$, aumentando el reclutamiento en las regiones pulmonares dependientes, optimizando la mecánica de la pared torácica, facilitando el drenaje de secreciones traqueobronquiales, aumentando la capacidad residual funcional y mejorando el flujo sanguíneo pulmonar ${ }^{(4)}$. Permitiendo de esta manera una distribución más homogénea y uniforme de la presión transpleural y la perfusión pulmonar ${ }^{(5)}$.

En un reporte de casos realizado por Sztajnbok $\mathrm{J}$ et $\mathrm{al}^{(6)}$ aplicó la posición en DP a dos pacientes con síntomas por COVID-19 que evolucionaron a SDRA, con requerimiento de oxígeno suplementario. Los pacientes se encontraban despiertos, sin intubación. Ambos pacientes informaron el alivio de la sintomatología respiratoria y presentaron una mejora en la $\mathrm{PaO} 2 / \mathrm{FiO2}$, que aumentó en $25-35 \mathrm{mmHg}$, lo que permitió disminuir el aporte de oxígeno. De esta manera se demostró que la posición en DP es una herramienta potencialmente útil para mejorar la oxigenación y el alivio de los síntomas.

El objetivo de este estudio de revisión narrativa es dar a conocer que la posición en DP presenta grandes beneficios que pueden implementarse hoy en día, en pacientes con infección por SARS-CoV-2 sin ventilación mecánica invasiva, con el objetivo de que sea utilizada no como una terapia de rescate, sino como una terapia para prevenir el deterioro de la función ventilatoria, pudiendo implementarse en todos los hospitales COVID.

\section{MECANISMO FISIOPATOLOGICO DEL SARS-COV-2 Y SU ASOCIACION AL SDRA}

El virus SARS-CoV-2 causante de la COVID-19 se introdujo a finales del 2019, propagándose rápidamente por todo el mundo y provocando un aumento en la tasa de mortalidad ${ }^{(7)}$. La COVID-19 puede variar desde un estado asintomático a una neumonía bilateral severa, causando la forma más grave de la enfermedad, el SDRA. La afectación pulmonar grave va a depender de la edad del paciente y la presencia de comorbilidades como la obesidad, hipertensión, diabetes mellitus (DM) y enfermedad renal, de los cuales 15\% de pacientes COVID-19 van a presentar daño pulmonar grave. Estudios refieren que la mortalidad estimada en promedio aumenta a $1,4 \%$ en pacientes $<60$ años y $4,5 \%$ en pacientes $>60$ años ${ }^{(8)}$.

Este virus causante del síndrome respiratorio agudo severo 2 (SARS-Cov-2), es un virus monocatenario con genoma ARN de cadena simple y positiva. La vía de transmisión principalmente se da por contacto entre personas y la diseminación de gotas respiratorias que producimos al hablar, toser o estornudar ${ }^{(9)}$. Se mencionan dos patrones causantes del daño pulmonar. El primero puede estar sujeto al efecto citopatico directo del virus, debido a la alta carga viral persistente en el tracto respiratorio superior e inferior. Y segundo, las altas concentraciones plasmáticas de citocinas proinflamatorias, sugiere una reacción inmunopatológica que causa daño pulmonar. Una vez que ha sido inhalado el virus del SARS-CoV-2, ingresa a las células del tracto respiratorio por medio del receptor de enzima convertidora de angiotensina 2 (ACE2), facilitando su adherencia en el tejido pulmonar, dando inicio a su replicación desde la mucosa oral , nasofaringe, neumocito II, hasta el endotelio vascular del pulmón ${ }^{(8)}$. Luego el virus se propaga y migra al tracto respiratorio a través de las vías aéreas conductoras, desencadenando una respuesta inmune innata. Aproximadamente el $80 \%$ de los pacientes infectados, desarrollan la forma leve de la enfermedad, limitándose principalmente a las vías aéreas superiores. Pero el $20 \%$ de los pacientes infectados progresan a las siguientes etapas, desarrollando infiltrados pulmonares y evolucionando a la forma grave de la enfermedad.(10)Hallazgos en tomografías computarizadas (TC) muestran el compromiso de ambos pulmones, donde se evidencian sombras irregulares bilaterales multifocales y opacidades en vidrio esmerilado, con una distribución pulmonar periférica ${ }^{(8)}$.

El virus llega a la unidad de intercambio de gases del pulmón, infectando preferentemente a las células alveolares tipo II, causando lesión en la pared alveolar y provocando la apoptosis y muerte de la célula. Debido a la pérdida de las células alveolares tipo Il se activa la vía secundaria para la regeneración epitelial, provocando daño difuso alveolar exudativo con congestión capilar masiva, principalmente en regiones periféricas y subpleurales, produciendo la cicatrización y fibrosis pulmonar, lo que conlleva a la perdida de la distensibilidad y disminución de la oxigenación pulmonar, iniciando como insuficiencia respiratoria aguda y evolucionando rápidamente a un SDRA ${ }^{(7)}$. Por lo 
tanto la lesión de las células epiteliales alveolares es la causa principal del SDRA en pacientes COVID-19 ${ }^{(10)}$.

El SDRA por COVID-19 se define como: lesión pulmonar inflamatoria aguda difusa, que conlleva al aumento de la permeabilidad vascular pulmonar, aumento del peso pulmonar y pérdida del tejido aireado, aumento del espacio muerto fisiológico y disminución de la distensibilidad pulmonar ${ }^{(2)}$. Hay que tener en cuenta que estudios realizados indican que el inicio del SDRA por COVID-19, no coincide con el inicio del SDRA según los criterios de Berlín. Según Huang y col. De 41 casos de pacientes COVID-19, la mediana de tiempo desde los primeros síntomas hasta el SDRA fue de 9 días $(8-14)^{111}$. Después, Wang et $\mathrm{al}^{(1)}$ de 138 casos de COVID-19 la mediana de tiempo desde el inicio de los síntomas hasta el SDRA fue de 8 días (6-12). Zhou y col. Dieron a conocer que la mediana de tiempo desde el inicio de la enfermedad hasta el SDRA fue de 12 días $(8-15)^{(12)}$. Debido a que el tiempo de inicio del SDRA relacionado con COVID-19 fue de 8 a 12 días, sugirió que el límite de inicio de 1 semana definido por los criterios SDRA de Berlín no se aplicaba para el inicio del SDRA en pacientes COVID-19. Es por eso que es importante identificar a tiempo el inicio de los síntomas, y poder dar un manejo oportuno, previniendo el rápido deterioro respiratorio del paciente $e^{(10)}$.

Con la pandemia global que ejerce presión sobre los recursos de muchos países, es urgente encontrar una maniobra de bajo riesgo y bajo costo para pacientes no intubados que detenga la progresión de la enfermedad ${ }^{(13)}$. Bien se sabe que la pandemia del COVID-19 ha llevado a un aumento en el ingreso de pacientes con insuficiencia respiratoria a los establecimientos de salud, agotando los recursos y la disponibilidad de las camas en $\mathrm{UCl}$, por lo que la gran parte de pacientes requieren soporte ventilatorio no invasivo, sin embargo, progresan a la fase crítica de la enfermedad rápidamente, siendo necesaria la intubación ${ }^{(14)}$. Debido a la gran carga laboral que presenta hoy en día el personal de salud se han puesto en práctica estrategias que previenen el deterioro respiratorio del paciente, dentro de ello tenemos la posición en DP, estrategia utilizada desde hace 4 décadas en pacientes con SDRA.

Se sabe que la posición en DP generalmente es utilizada en pacientes con SDRA moderado a severo con VMI, esta posición promueve la homogeneidad pulmonar, mejorando el intercambio de gases y la mecánica respiratoria, lo que permite reducir la intensidad de la ventilación y reducir la lesión pulmonar; método recomendado por guías basadas en evidencia. Sin embargo, hoy en día existen diversos estudios que demuestra que el uso temprano de la posición en DP mejora la oxigenación y previene la intubación en pacientes con infección por SARS-CoV-2 sin $\mathrm{VMI}^{(4)}$.

Diversos estudios demuestran que la posición en decúbito prono, presenta un gran beneficio en la mejora de la oxigenación en pacientes con SDRA que se encuentran con o sin VM ${ }^{(15)}$. Desde hace cuatro décadas se han establecido beneficios de la posición en decúbito prono en pacientes con SDRA, mejorando la relación ventilación/perfusión (V/Q), aumentando el reclutamiento en las regiones pulmonares dependientes, optimizando la mecánica de la pared torácica y facilitando el drenaje de secreciones traqueobronquiales ${ }^{(16,17)}$. La gravedad y la compresión de las estructuras mediastínicos y abdominales contribuyen al cambio de la perfusión y la ventilación del tejido pulmonar. Se han encontrado avances de estudios que han permitido obtener imágenes por medio de resonancia sobre los cambios posturales en el flujo sanguíneo pulmonar y el efecto del corazón sobre la perfusión pulmonar en respuesta a la pronación ${ }^{(4)}$. Se ha evidenciado, que tanto en pacientes con SDRA por COVID-19 con VM presentan el mismo mecanismo fisiopatologico que en pacientes con SDRA por COVID-19 $\sin \mathrm{VM}^{(18)}$.

\section{MECANISMOS FISIOPATOLOGICOS DE LA POSICION EN DP}

Normalmente en un pulmón sano y en decúbito supino (DS) el desplazamiento de los gases está dado por una gradiente gravitacional de presión pleural necesaria para mantener la distensión pulmonar. Estudios tomográficos demostraron que la posición en DP induce a un mayor reclutamiento pulmonar y la reducción de la sobredistensión pulmonar ${ }^{(15,19)}$. Las estructuras de las vías respiratorias y los vasos sanguíneos pulmonares presentan asimetrías en los diámetros de ramificación y ángulos, lo que resulta en una distribución heterogénea en la ventilación y perfusión ${ }^{(20)}$.

\section{Disminución de la gradiente gravitacional en la presión pleural.}

La ventilación del pulmón en DS en presencia de SDRA presenta una distribución heterogénea, no uniforme. Los alveolos ventrales se dilatan con facilidad a diferencia de los alveolos dorsales que se encuentran relativamente comprimidos por el peso del corazón y el mediastino, por compresión del lóbulo inferior izquierdo. La gravedad y la forma triangular del pulmón que se expande dentro de la caja torácica (cofre esférico) durante la inspiración dan como resultado una distribución heterogénea de las presiones transpulmonares a través del eje ventral-dorsal ${ }^{(18)}$. Lo que quiere decir que la presión que aumenta de la región ventral a la dorsal, contribuye a las diferencias en la distribución de la densidad en todo el pulmón, dando como resultado la disminución del tamaño alveolar en posición supina que en posición prona, provocando una mayor expansión en las regiones no dependientes y menor expansión en las regiones dependientes ${ }^{(21)}$. 
Se sabe que la presión transpulmonar es la diferencia entre la presión alveolar y la presión intrapleural, por tal motivo, cuando se produce un aumento de la presión transpulmonar, la expansión pulmonar y la entrada de aire es mayor. En DS dicha presión transpulmonar es mayor en las regiones no dependientes que en las regiones dependientes ${ }^{(5)}$. En un estudio realizado por West, plantea la "hipótesis gravitacional", donde en un pulmón sano en posición vertical el cociente ventilación-perfusión disminuye desde el vértice a la base, pero, si existe afectación, se observa una desorganización del patrón regular, finalizando en la alteración a nivel alveolar de la relación normal entre la ventilación y el flujo sanguíneo ${ }^{(15,19)}$. Si tomamos esta teoría gravitacional en pacientes en DP se observaría un gradiente no gravitacional de distribución de la perfusión pulmonar de las regiones no dependientes a las regiones dependientes del pulmón, favoreciendo el incremento de la $\mathrm{PaO} 2$ y mejorando la relación $\mathrm{V} / \mathrm{Q}$, lo que genera una ventilación alveolar más uniforme $y$ homogénea, al realizar el cambio de DS a DP. Estudios experimentales demostraron que la presión pleural en DP se encontraba reducida significativamente a lo largo del eje anteroposterior en relación con el DS, lo que favorecía una distribución más homogénea y uniforme de la presión transpleural y la perfusión pulmonar ${ }^{(5)}$.

\section{Cambios en la motilidad del diafragma.}

La posición en DP tiene como base teórica el modelo de los compartimentos torácico y abdominal, que contienen órganos de diferentes densidades, separados por el diafragma, presentando un volumen abdominal de $10 \mathrm{~L}$ en comparación del volumen torácico de $5 \mathrm{~L}$. La cavidad abdominal presenta 2 paredes rígidas sujetado por la pelvis y la columna vertebral, y dos paredes flexibles compuesta por la superficie ventral y el diafragma. Tanto la presión pleural como la presión abdominal cambian la desviación de las paredes flexibles de la pared abdominal y el cambio de posición del cuerpo, lo que influye en la forma y posición del diafragma. La presión hidrostática de la cavidad torácica es menor que las presiones de la cavidad abdominal en DS. Asimismo, la gradiente vertical de la presión intrabadominal influye sobre la gradiente vertical de la presión pleural en DS ${ }^{(20)}$.

Debido a la presencia de la columna vertebral y los músculos paravertebrales, la región dorsal es más rígida que la región ventral y tanto la presión pleural como la presión intraabdominal se modifican al cambio de posición. Los pacientes en DS presentan una menor expansión diafragmática durante la inspiración, lo que favorece la formación de atelectasias dorsales en DS, en cambio, cuando se coloca al paciente en DP, la expansión torácica principalmente se produce en la región abdominal y dorsal, influenciada por el incremento de la rigidez de la pared abdominal, favoreciendo una mejor oxigenación y relación $\mathrm{V} / \mathrm{Q}$ en las zonas más declives ${ }^{(15,19)}$.

\section{Efectos del peso y tamaño del corazón}

Normalmente en DS el corazón y las estructuras mediastínicas descansan sobre el volumen pulmonar y en DP la estructura cardiaca descansa sobre el esternón. En un paciente con SDRA ocurre un aumento del tamaño y el peso del corazón debido al edema en las paredes cardiacas y dilatación del ventrículo derecho por la hipertensión pulmonar, lo que llevaría a una mayor compresión del volumen pulmonar, de tal forma que la compresión de la estructura cardiaca sobre el lóbulo inferior izquierdo en DS conlleva a la disminución de la oxigenación ${ }^{(15)}$. Entonces, cuando se realiza el cambio de DS a DP, la atelectasia causada por compresión de la estructura cardiaca y el mediastino, produce la disminución de las fuerzas de distensión de los alveolos ventrales, dando lugar a una distribución ventilatoria más homogénea. Además, la perfusión pulmonar mejora considerablemente en las regiones ventrales debido a la mayor producción de óxido nítrico, un potente vasodilatador que modifica el mecanismo vasoconstrictivo ${ }^{(18)}$.

Se observó la mejoría en la oxigenación pulmonar en 31 estudios observacionales, de los cuales $20 \%$ de los estudios informaron una respuesta positiva de la oxigenación en el $70 \%$ de los sujetos, mientras que el 47\% informaron una respuesta positiva en el $70-85 \%$ de los sujetos y el $33 \%$ de los estudios, encontró mejor oxigenación en el $90 \%$ aproximadamente. Se ha evidenciado en la mayoría de los estudios el aumento de la $\mathrm{PaO} 2 / \mathrm{FiO} 2$ con valores de $10-20 \mathrm{mmHg}$ o un aumento del $10 \%$ a $20 \%^{(20)}$.

\section{Efectos del volumen pulmonar}

Debido a que la pared torácica dorsal es menos flexible que la pared torácica ventral, el efecto al realizar el DP, es disminuir el cumplimiento general de la pared torácica. La disminución del cumplimiento de la caja torácica durante el DP, disminuye la redistribución de la densidad pulmonar de las áreas dorsales a ventrales, aumentando la oxigenación en las regiones dorsales atelectasiadas, mejorando el intercambio de gases ${ }^{(21)}$.

En DS un 50\% del tejido pulmonar se encuentra orientada hacia el dorso del tórax, mientras que un $20 \%$ se encuentra orientada en el plano ventral, debajo del pulmón. Es por eso que la distribución del tejido pulmonar dentro de la caja torácica, explica la gran relevancia clínica de la posición en DP en comparación a la posición en $\mathrm{DS}^{(20)}$. El porcentaje del volumen pulmonar debajo del corazón en personas sanas en DS abarca el $40 \%$ del hemitórax izquierdo mientras que en DP el volumen pulmonar no llega al $4 \%$. En pacientes con SDRA fisiológicamente presentan un aumento del peso pulmonar debido al incremento de la presión 
hidrostática, lo que es 4 a 5 veces superior a lo normal, el tejido pulmonar se vuelve más rígido y el cumplimiento de la caja torácica disminuye en asociación con la compresión, originando el colapso de las regiones declives o dependientes (atelectasia por compresión) y aumentando la distención de las regiones no declives por tracción alveolar ${ }^{(19)}$. El cambio de posición de DS a DP según estudios, aumenta el volumen pulmonar, en un $17 \%$. Las gradientes gravitacionales se reducen, mejorando la perfusión y ventilación del parénquima pulmonar. Algunos estudios han encontrado un aumento en la perfusión global en posición prona y una distribución más homogénea, debido a una mayor capacidad residual funcional y un gradiente gravitacional reducido ${ }^{(4)}$. En 1976 Piehl y Browm, refirieron que al colocar a los pacientes con insuficiencia respiratoria en DP, mejoraba la oxigenación, esto fue respaldado en 1977 por Douglas. Posteriormente en 1986, Beck y Rehder, realizaron una investigación experimental en perros, demostrando que la conductancia vascular fue mayor en la región dorsal que en la región ventral del pulmón ${ }^{(22)}$. Pelosi y col. Demostraron que la variación del cumplimiento de la pared torácica está reflejada en la $\mathrm{PaO} 2 / \mathrm{FiO2}^{(23)}$.

Bien se sabe que el brote de la enfermedad por COVID19 es una infección respiratoria que puede empeorar rápidamente a insuficiencia respiratoria hipoxémica grave y síndrome de dificultad respiratoria aguda (SDRA) en algunos pacientes. Por lo que el $40 \%-80 \%$ de pacientes con SDRA severa requieren ventilación mecánica invasiva (VMI), lo que conduce a un aumento en la tasa de mortalidad. Por lo tanto, se están realizando esfuerzos significativos para apoyar la función respiratoria del paciente y evitar el uso de ventilación mecánica invasiva ${ }^{(4)}$. Hoy en día se postula que la posición en DP en pacientes con infección por SARS- CoV-2 no intubados es igual de beneficioso que en pacientes intubados. En un estudio con 20 pacientes con SDRA sin VMI, se demostró que el $55 \%$ de los pacientes, no requirieron intubación al final del estudio, incluyendo a los pacientes con SDRA moderado severo ${ }^{(24)}$.

\section{BENEFICIOS DEL DECUBITO PRONO EN PACIENTES CON INFECCION POR SARS-COV-2 SIN VENTILACION MECANICAINVASIVA}

En 1976 por primera vez se implementó la posición en DP para mejorar la oxigenación en pacientes con SDRA que se encontraban en $\mathrm{UCl}$ con ventilación mecánica. Diferentes estudios demostraron que en DS durante la inspiración existe una distribución difusa de la oxigenación pulmonar, la distribución del aire se dirige a las regiones no dependientes, debido al colapso alveolar en las regiones dependientes, que vendrían a ser las menos oxigenadas, lo que provoca un desequilibrio en la relación $\mathrm{V} / \mathrm{Q}$. Mientras que en DP, la disponibilidad del parénquima pulmonar aumenta, dándose un mayor reclutamiento alveolar, disminuyendo la deformación de las fibras y la tensión pulmonar ${ }^{(19)}$. Sin embargo existen estudios que abalan el beneficio del posicionamiento en prono para evitar el deterioro respiratorio del paciente con infección por SARS-CoV-2 que se encuentran sin VM y prevenir la intubación. Algunos investigadores han informado que la posición en DP mejora la oxigenación en pacientes no intubados tratados con oxigenoterapia estándar, presión positiva continua (CPAP) o ventilación no invasiva. El beneficio es que reduce la lesión pulmonar autoinducida. Por lo que disminuye la necesidad de intubación y el posterior ingreso a la $\mathrm{UCl}^{(14)}$.

Se ha postulado que adoptar la posición en DP para pacientes no intubados con COVID-19 que requieren soporte respiratorio básico es tan beneficioso como en pacientes con SDRA con VM, pudiendo mejorar en ambos la oxigenación, al reducir la necesidad de ventilación invasiva y la mortalidad ${ }^{(25)}$. En un reporte de casos realizado por Sztajnbok J et $a^{(6)}$ se aplicó la posición en DP a dos pacientes con síntomas COVID-19 que evolucionaron a SDRA, con oxígeno suplementario con mascara de reservorio a $10 \mathrm{~L}$. Los pacientes se encontraban despiertos, sin intubación. Ambos pacientes informaron el alivio de la sintomatología respiratoria y presentaron una mejora en la $\mathrm{PaO} 2 / \mathrm{FiO2}$, con aumento en $25-35 \mathrm{mmHg}$ en cada uno de los pacientes, lo que permitió disminuir el aporte de oxígeno, de $10 \mathrm{~L}$ a $5 \mathrm{~L}$. De esta manera, se demostró que la posición en DP es una herramienta potencialmente útil para mejorar la oxigenación y el alivio de los síntomas (Tabla 1) ${ }^{(6)}$.

Scaravilli y colegas realizaron un estudio retrospectivo con 15 pacientes no intubados, con insuficiencia respiratoria aguda (IRA) hipoxemica. Se sometieron a 43 sesiones en DP, y refirieron que la posición es factible en el $95 \%$ de las sesiones realizadas y un aumento significativos de la $\mathrm{PaO} 2$ hasta 6 horas después de la resupinación. Sin embargo, este estudio está limitado por el pequeño número de pacientes y la naturaleza retrospectiva del estudio (Tabla 1$)^{(26)}$.

Con base en la evidencia de guías de atención del paciente crítico y estudios realizados con anterioridad, se creó un protocolo multiinstitucional en el Hospital New York-Presbyterian para el manejo de pacientes despiertos, no intubados, con COVID-19 presuntivo o confirmado del departamento de emergencia. Los criterios de inclusión incluían pacientes: COVID-19, confirmados o sospechados, requerimiento de $\mathrm{O} 2>4 \mathrm{~L}$, en camilla, con monitor de oximetría de pulso continuo, despierto, capaz de seguir instrucciones, capaz de tolerar cambios de posición y capaz de realizar el cambio de posición con asistencia mínima. Los criterios de exclusión incluían pacientes con: saturación normal de oxígeno, sin necesidad de oxígeno suplementario, estado mental alterado, incapacidad de tolerar o 
cambiar independientemente de posición, inestabilidad hemodinámica, incapacidad para seguir instrucciones. Se coloca al paciente en DP en la camilla en trendelenburg levemente inverso. Los pacientes realizaban el cambio de posición cada 30 min según sea tolerado y durante el mayor tiempo posible. Fueron reevaluados cada 30 min durante la primera hora y cada hora durante las próximas 2 horas. Se mostró que más de 600 pacientes con COVID-19 despiertos, sin intubación ,presentaron efectos significativos para mejorar la oxigenación y la heterogeneidad pulmonar $^{(24)}$.

Recientemente, se informó que el DP mejora la oxigenación y la capacidad de reclutamiento pulmonar cuando se combina con ventilación no invasiva en pacientes con SARS por COVID-19. Se realizó un estudio retrospectivo observacional en tres hospitales en las ciudades de Wuhu y Maanshan en el 2020, donde fueron examinados 79 pacientes COVID-19, despiertos. Diez fueron graves y todos fueron colocados en DP, en combinación con tratamiento con cánula nasal de alto flujo (CNAF). La gravedad de la enfermedad se calificó de acuerdo con las pautas para el diagnóstico y el tratamiento de la infección por el nuevo coronavirus, por la Comisión Nacional de Salud. El tiempo objetivo de posicionamiento en DP fue más de $16 \mathrm{~h}$ al día, con cambio de posición cada 2 horas, pudiendo adecuarse de acuerdo a la tolerancia del paciente. La mediana $\mathrm{PaO2} / \mathrm{FiO} 2$ se elevó significativamente después de DP. Ninguno de los pacientes progresó a un estado crítica o necesitó intubación endotraqueal. Se demostró que el aporte de oxígeno con CNAF en pacientes COVID-19 combinada con posición en DP podría usarse de manera segura y eficaz en pacientes graves con COVID-19, reduciendo la conversión al estado crítico de la enfermedad y la necesidad de intubación (Tabla 1).(27) Otros estudios realizados, aplicaron la posición en DP en paciente despiertos, no intubados con SDRA moderado a severo combinado con oxígeno suplementario con CNAF, se demostró que la $\mathrm{PaO} 2 / \mathrm{FiO} 2$ aumento de 25 a $35 \mathrm{mmHg}$, evitando que la mitad de los pacientes sean intubados. Solamente se sabe de dos informes y una serie de casos, donde 50 pacientes COVID-19, no intubados, fueron evaluados, con una SpO2 de $80 \%$ que aumentó a $84 \%$ al colocarle oxígeno suplementario, al realizar el DP, después de 5 minutos, la saturación aumentó significativamente a 94\%; 13 pacientes no respondieron al DP, por lo que se requirió la intubación dentro de las 24 horas de su llegada a emergencia, demostrándose que una gran cantidad de pacientes respondieron a la posición en $\mathrm{DP}^{(6)}$.

Se realizó un estudio prospectivo en pacientes COVID19, despiertos, no intubados. Los pacientes fueron ingresados en el Hospital Aix-en-Provence, Francia, 2020, por insuficiencia respiratoria aguda hipoxemia que requerían suplemento de oxígeno. Fueron considerados solo pacientes COVID-19 confirmados que requerían oxígeno suplementario y tenían hallazgos tomográficos sugestivos de COVID-19 con lesiones posteriores. Se realizó el seguimiento durante 10 días. Se tomó como criterios de exclusión pacientes con insuficiencia respiratoria aguda que requiere intubación y alteración de la conciencia. Se midieron los gases en sangre arterial, antes de DP, durante DP y 6 a 12 horas después de la resupinación. Fueron 24 pacientes que participaron en el estudio, de los cuales, 4 no toleraron DP más de 1 hora (17\%), 5 toleraron durante 1 a 3 horas (21\%) y 15 toleraron durante más de 3 horas, (63\%). Aumentó la $\mathrm{PaO} 2 \geq 20 \%$ durante DP y después de la resupinación. Sin embargo, la oxigenación aumentó durante el DP en solo un $25 \%$ y no se mantuvo en la mitad de las personas después de la resupinación, 4 pacientes requirieron intubación dentro de las 72 horas. A pesar de que la mayor cantidad de pacientes toleraron más de 3 horas el DP, no lograron mantener una saturación después de regresar a la posición a DS, sin embargo la cantidad de pacientes intubados, fue mínima (Tabla 1$)^{(28)}$.

Según Ding et $a^{(25)}$ concluye que la aplicación temprana de posicionamiento prono con cánula nasal de alto flujo, especialmente en pacientes con SDRA moderado y $\mathrm{SpO} 2$ basal> 95\%, puede ayudar a mejora de manera considerable la oxigenación. Los estudios mencionados, nos dan a conocer, que a pesar de que la posición en DP fue utilizada desde un inicio en pacientes con SDRA con VM como efecto protector a la lesión pulmonar, bien podemos darnos cuenta que dicha estrategia hoy en día, nos puede ayudar a revertir el deterioro del sistema respiratorio en un paciente con infección por SARS-CoV-2 sin VM por COVID-19 en etapas iniciales de la enfermedad, evitando la intubación y el ingreso a $\mathrm{UCl}$.

Así como existen estudios que demuestran el beneficio de la posición en DP en pacientes con infección por SARS-CoV-2 sin VMI, por otro lado, existen estudios que no corroboran el beneficio de este posicionamiento.

En un estudio cohorte prospectivo, se seleccionaron 56 pacientes con neumonía, despiertos, no intubados, con edad media de 57 años, con diagnostico confirmado de neumonía por COVID-19, con oxígeno suplementario o presión positiva continua no invasiva. Se realizó DP mantenido, durante un mínimo 3 horas. La posición en DP fue factible y se mantuvo por 3 horas continuas en 47 pacientes $(83,9 \%)$. La oxigenación mejoró por un aumento de $\mathrm{PaO} 2 / \mathrm{FiO} 2$ de $180.5 \mathrm{mmHg}$ en posición supina a un $\mathrm{PaO} 2 / \mathrm{FiO} 2$ de $288.5 \mathrm{mmHg}$ en posición prono, 23 pacientes presentaron mejora de la $\mathrm{PaO} 2 / \mathrm{FiO} 2$ de $192,9 \mathrm{mmHg}$ 1hora después de la resupinación. Al final del estudio se concluyó que $13(28 \%)$ de 46 pacientes fueron intubados, 7(30\%) de 23 eran respondedores y $6(26 \%)$ de 23 eran no respondedores. $\mathrm{La} \mathrm{PaO2/FiO2} \mathrm{aumento} \mathrm{en} \mathrm{un} 10$ a $20 \%$ en pacientes respondedores. En este estudio 
prospectivo observacional se demostró, que no hubo significancia en la tasa de intubación entre los respondedores y no respondedores. Este estudio respalda la viabilidad del DP en pacientes despiertos, con ventilación espontanea. Sin embargo, en este estudio también muestran la ausencia de una mejora en la oxigenación duradera. Los datos de este estudio sugieren que la posición en DP de manera temprana y en pacientes con marcadores inflamatorios aumentados es más beneficiosa, lo que es explicado por un mayor reclutamiento en la primera fase del SDRA o por una redistribución de la perfusión más persistente. En el estudio, el posicionamiento en DP no demostró mejora a largo plazo, sin embargo, podría retrasar o evitar la intubación (Tabla 1$)^{(14)}$.

Si se analiza detalladamente el estudio de Scaravilli et $\mathrm{al}^{(26)}$ de los 15 pacientes, 2 pacientes de estar recibiendo oxígeno con CNAF y casco receptor (CPAP), fueron cambiados a ventilación por presión positiva no invasiva, a pesar de presentar un aumento en la $\mathrm{PaO2} / \mathrm{FiO2}$. Por lo que se considera como un fracaso al tratamiento. En este mismo estudio refieren que 3 pacientes murieron después del DP y 2 murieron después de la intubación, porque se considera que 3 de los pacientes murieron por no haber sido intubados a tiempo, lo que conduce a una mayor mortalidad. Es por eso que no podemos decir de manera certera, que la posición en DP es beneficiosa para pacientes con hipoxemia leve a moderada. Generalmente la posición en DP se utiliza como una maniobra de rescate en pacientes conectados a VMI, sin embargo, su uso en pacientes con hipoxemia leve a moderada aún sigue siendo controvertido. Hay que tener en cuenta que los últimos estudios concluyen que la duración del DP da resultados beneficiosos y el inicio temprano como se menciona en el estudio de Scaravilli mejora la oxigenación al facilitar la movilización de secreciones de las vías respiratorias y revertir la atelectasia que se encuentra más comprometida en el lado izquierdo debido a la masa del corazón. Sin embargo, los efectos mencionados son de corta duración ${ }^{(26)}$.

En un estudio retrospectivo, se incluyeron 10 pacientes con prueba confirmada para SARS-CoV-2, que requerían oxígeno suplementario con colocación de cánula binasal. Se les realizo seguimiento durante 28 días del alta hospitalaria. Se realizó el posicionamiento en DP cada 2 horas y durante la noche según sea tolerado. Antes de la colocación en DP, el requerimiento de oxígeno era del $40 \%$. La oxigenación aumento al realizar la posición al cabo de 1 hora, aumentando la saturación de oxígeno de $94 \%$ a $98 \%$ y una disminución de la frecuencia respiratoria. Dentro de los resultados primarios se encontró cambios en la saturación de oxígeno y de la frecuencia respiratoria al cabo de 1 hora. Se demostró que 5 de 6 pacientes que se encontraban con cánula nasal, no requirieron intensificación de soporte ventilatorio y 8 de los 10 pacientes no requirieron ventilación mecánica invasiva. Se debe tener en cuenta que existieron limitaciones al realizar el estudio, la muestra fue pequeña y no se llevó a cabo un control. Si bien el estudio realizado, nos muestra el beneficio de la posición en DP casi en la totalidad de los pacientes, aún quedan dudas por resolver, no se sabe si el efecto de la posición en DP continua después de la supinación, o si en realidad prevenir evita la necesidad de la intubación o solo lo retrasa, tampoco sabemos si los pacientes se pudieron haber recuperado sin la intervención del DP. Aún son muchas las dudas que se tienen que resolver y muchos los estudios que se tienen que llevar a cabo (Tabla1) $^{(29)}$.

\section{Duración del decúbito prono}

Estudios refieren que la posición en DP es más efectiva cuando su inicio es temprano, dentro de los 3 días de iniciado el SDRA, cuando las atelectasias están congestivas y compresivas, en relación con la fase intermedia donde es más prevalente la fibrosis y la hiperplasia de las células tipo II. Sin embargo, varios estudios refieren que existe mejora de la oxigenación pulmonar cuando se inicia la posición en DP a los 6 a 11 días de iniciado el SDRA.(20)Recientemente, se ha demostrado que el reclutamiento de zonas pulmonares posteriores depende de la duración del posicionamiento $^{(30)}$.

La duración durante el DP para mejorar la oxigenación es muy variable. Sin embargo, la respuesta típica se da los 30 minutos de realizar la posición, seguido de una respuesta continua de 24 horas $^{(18)}$. Langer et al(31) informó que la $\mathrm{PaO} 2$ aumentó de 70 a $90 \mathrm{mmHg}$ a los 30 min y alcanzó $112 \mathrm{mmHg}$ a las $2 \mathrm{~h}$ en pacientes con SDRA moderado. Papazian et $\mathrm{al}^{(32)}$ informó que el $73 \%$ de pacientes en DP presentaron una mejora rápida de la $\mathrm{PaO2} / \mathrm{FiO2}$ al cabo de 1 hora, mientras que el 27\% fueron respondedores lentos, que requieren $6 \mathrm{~h}$ para evidenciar mejora en la $\mathrm{PaO2} / \mathrm{FiO2}$. Pappert et al(33) informó resultados similares, con $\mathrm{PaO} 2 / \mathrm{FiO} 2$ mejorando de 98 a $136 \mathrm{mmHg}$ a los $30 \mathrm{~min}$ a $146 \mathrm{mmHg}$ a las $2 \mathrm{~h}$ en pacientes con SDRA. Existen estudios que indican el beneficio de las sesiones prolongada de más de $48 \mathrm{~h}$ en DP. Romero et $\mathrm{al}^{\left({ }^{(34)}\right.}$ mantuvo al paciente en DP durante $55 \mathrm{~h}$, a las $48 \mathrm{~h}$ se informó la mejora de la $\mathrm{PaO} 2 / \mathrm{FiO} 2$ ( $92 \mathrm{mmHg}$ frente a $227 \mathrm{mmHg}$ ) en pacientes con SDRA moderado, sin VM. Además hay evidencia de que los pacientes con SDRA, no intubados, no respondedores iniciales a DP pueden convertirse en respondedores en intentos posteriores. Lo que nos da a entender que a más repeticiones y mayor duración, mayor es la probabilidad de que exista una mejor oxigenación ${ }^{(20)}$.

Hoy en día, debido a que las unidades de cuidados intensivos $(\mathrm{UCl})$ se encuentran saturadas, aplicar la posición en DP, puede evitar la progresión del paciente 
Tabla 1. Estudios realizados en posición prono en pacientes con infección por SARS-CoV-2 sin ventilación mecánica invasiva

\begin{tabular}{|c|c|c|c|c|c|}
\hline Autor & Pais & Tipó de estudio & Prono, tiempo & Tipo de vni & Conclusion \\
\hline $\begin{array}{l}\text { Jaques } \\
\text { Sztajnbok } \\
\text { et al(6) }\end{array}$ & Brasil & Reporte de casos & $\begin{array}{l}\text { Paciente } 1 \\
10 \text { horas } \\
\text { Paciente } 2 \\
8 \text { horas }\end{array}$ & Mascara de reservorio & $\begin{array}{l}\text { Mejora de los síntomas. } \\
\text { Reducción de la necesidad de oxígeno, de } 10 \mathrm{~L} \text { a } 5 \mathrm{~L} \text {. } \\
\text { Ningún paciente requirió intubación }\end{array}$ \\
\hline $\begin{array}{l}\text { Scaravilli } \\
\text { Vittorio et } \\
\text { al(26) }\end{array}$ & Italia & Estudio retrospectivo & $\begin{array}{l}\text { Mediana de ciclos: } 2 \\
\text { sesiones } \\
\text { Mediana de duración: } 3 \\
\text { horas }(2-4 \mathrm{~h})\end{array}$ & Ventilación espontanea & $\begin{array}{l}\text { El DP fue factible, seguro y con un significativo beneficio en la } \\
\text { oxigenación }\end{array}$ \\
\hline $\begin{array}{l}\text { Qiancheng } \\
\text { Xu et al(27) }\end{array}$ & China & Estudio retrospectivo & $\begin{array}{l}\text { Tiempo objetivo } 16 \\
\text { horas } \\
\text { Cambio de posición cada } \\
2 \text { horas }\end{array}$ & CNAF & $\begin{array}{l}\text { El uso temprano del DP combinado con HFNC mejora la } \\
\text { oxigenación y evita la intubación. } \\
\text { Ningún paciente fue intubado }\end{array}$ \\
\hline $\begin{array}{l}\text { Xavier } \\
\text { Elharrar et } \\
\text { al(28) }\end{array}$ & Francia & Estudio prospectivo & $\begin{array}{l}\text { Tiempo mayor en DP: } 3 \\
\text { horas }\end{array}$ & CNAF & $\begin{array}{l}\text { La oxigenación aumentó durante el DP en solo un } 25 \% \text { y no se } \\
\text { mantuvo en la mitad de las personas después de la } \\
\text { resupinación. } \\
4 \text { pacientes fueron intubados }\end{array}$ \\
\hline $\begin{array}{l}\text { Anna Coppo } \\
\text { et al (14) }\end{array}$ & Italia & Estudio cohorte prospectivo & $\begin{array}{l}\text { Tiempo mínimo de } 3 \\
\text { horas }\end{array}$ & CNAF & $\begin{array}{l}\text { Aumento en la relación } \mathrm{PaO} 2 / \mathrm{FiO} \text {. } \\
\text { El posicionamiento fue factible y efectivo para mejorar la } \\
\text { oxigenación. } \\
\text { El efecto se mantuvo en la mitad de pacientes después de la } \\
\text { resupinación }\end{array}$ \\
\hline $\begin{array}{l}\text { Mahendra } \\
\text { Damarla et } \\
\text { al(29) }\end{array}$ & EE.UU & Estudio retrospectivo & $\begin{array}{l}\text { Mediana de tiempo : } 5 \\
\text { horas }\end{array}$ & CNAF & $\begin{array}{l}\text { La oxigenación aumento al realizar la posición al cabo de } 1 \\
\text { hora, aumentando la saturación de oxígeno de } 94 \% \text { a } 98 \%\end{array}$ \\
\hline
\end{tabular}

al estado crítico y su ingreso a $\mathrm{UCI}^{(28)}$.

La Sociedad de Cuidados Intensivos del Reino Unido manifestó recientemente por un ensayo de posicionamiento DP en pacientes conscientes con COVID-19 confirmado o sospechoso y una FiO2 $\geq 28 \%$ para mantener $\mathrm{SpO2} 92-96 \%$ o $88-92 \%$ en aquellos con riesgo de insuficiencia respiratoria hipercapnica. Sugieren utilizar ciclos de 30 minutos a 2 horas, rotando al DP por el lado derecho y al supino por el lado izquierdo. Resultando en una mejora en la oxigenación. Sin embargo, aún no se ha evaluado completamente la efectividad y la duración óptima ${ }^{(18)}$.

VNI, ventilación mecánica no invasiva; CNAF, cánula nasal de alto flujo; DP, decúbito prono; EE.UU, Estados Unidos.

\section{Mortalidad}

A medida que se realizan prácticas modernas de ventilación junto con pacientes con SDRA grave, surgió evidencia de que la aplicación temprana en DP prolongado puede disminuir significativamente la mortalidad en comparación con la ventilación supina convencional ${ }^{(25)}$. Guerin et $\mathrm{al}^{(35)}$ demostraron que la aplicación de sesiones prolongadas de aproximadamente 17 horas durante 4 días en posición en DP en pacientes con SDRA grave, reducía el riesgo absoluto de la mortalidad en un $17 \%$ y el riesgo relativo en un $50 \%$. Sin embargo, existen otros estudios que no demuestran los beneficios de la posición sobre la mortalidad. El estudio PROSEVA demostró que la ventilación prolongada en posición en DP disminuyó considerablemente la mortalidad a los 28 días en un $16 \%$ y a los 90 días en un $16 \%$ en relación a la posición en DS en pacientes con SDRA ${ }^{(36)}$.

Se realizó la posición en DP seleccionando a 10 pacientes al azar que presentaban COVID-19, 70\% hombres y $30 \%$ mujeres, con una edad media de 41 años, 30\% de los pacientes presentaban comorbilidades (diabetes o hipertensión). Fueron hospitalizados en una sala no UCI sin intubación para pacientes COVID-19. Se observó que la Sp02\% promedio fue de $85,6 \%$ antes del DP y $95,9 \%$ después del posicionamiento. Se observó que la SpO2\% aumentó considerablemente. Además, la sensación de disnea disminuyó en el $40 \%$ de pacientes. Los pacientes fueron hospitalización por 4,8 días y no se produjeron muertes. Todos los pacientes fueron dados de alta. Los resultados mostraron clínicamente la mejoría del estado respiratorio y la $\mathrm{SpO} 2 \%$ en pacientes COVID-19 en estadio leve de la enfermedad y reducción de la mortalidad. En este estudio se observó que la posición en DP puede ayudar a pacientes COVID-19 que sufren la forma leve de la enfermedad. Sin embargo, aún se necesita más estudios para determinar el efecto protector de este posicionamiento ${ }^{(16)}$.

En 5 ensayos realizados en los últimos 15 años, al comparar la posición en DP con DS, se obtuvieron resultados sobre la supervivencia. Llegando a la conclusión, que la mortalidad tuvo una reducción significativa solo en pacientes con hipoxemia severa ( $\mathrm{PaO} 2 / \mathrm{FiO2}<100 \mathrm{mmHg}$ ) demostrado por un metaanálisis de datos de pacientes. Lo que fue confirmado por un ensayo con pacientes con SDRA moderado a severo $(\mathrm{PaO} 2 / \mathrm{FiO} 2<150 \mathrm{mmHg}$ y PEEP de 5 $\mathrm{cmH} 2 \mathrm{O}$ o más y FiO2 de 0,6 y por ultimo un metaanálisis 
dio a conocer que el beneficio del DP se asoció a la duración de sesiones y la intensidad de la hipoxemia ${ }^{(37)}$.

A pesar de que los estudios evidencian la disminución de la mortalidad al realizar el posicionamiento prono, aun se requieren más estudios que abalen la intervención de este posicionamiento sobre la tasa de mortalidad en pacientes con infección por SARS-CoV-2 que no se encuentran en VMI. Sin embargo, podríamos estar frente a una gran estrategia que hoy por hoy puede contribuir en la mejora del paciente con infección por SARS-CoV-2, evitando el progreso al estado crítico de la enfermedad y sobre todo previniendo o retrasando la intubación y el ingreso a UCl.

\section{CONCLUSION}

Se ha demostrado que la posición en DP en pacientes con SDRA con VMI presenta el mismo mecanismo fisiopatológico que en pacientes con SDRA por COVID-19 sin VMI.

Se ha postulado que adoptar la posición en DP para pacientes no intubados con COVID-19 que requieren soporte respiratorio básico es tan beneficiosa como en pacientes con SDRA con VMI, pudiendo mejorar en ambos la oxigenación, al reducir la necesidad de ventilación invasiva.

El cambio de posición de DS a DP, reduce las gradientes gravitacionales, mejorando la perfusión y ventilación del parénquima pulmonar.

La posición en DP en pacientes con SDRA por COVID-19, promueve la homogeneidad pulmonar, mejorando el intercambio de gases y optimizando la mecánica respiratoria.

Si bien los estudios evidencien disminución de la mortalidad al realizar el DP, aún se requieren más evidencia que abale la intervención de este posicionamiento sobre la tasa de mortalidad en pacientes con infección por SARS-CoV-2 que no se encuentran con VMI.

Se ha demostrado que el DP en pacientes despiertos, con respiración espontánea, no intubados con COVID19 , es factible, segura y está asociada a un beneficio significativo sobre la oxigenación. Si bien es necesario realizar más estudios, con este articulo narrativo esperamos fomentar el uso de la posición prona en pacientes despiertos con COVID-19, como un tratamiento no invasivo que puede prevenir el empeoramiento de la hipoxemia.

Conflictos de interés: Los autores niegan conflictos de interés.

Financiamiento: Autofinanciado.

\section{REFERENCIAS BIBLIOGRÁFICAS}

1. Wang, D., Hu, B., Hu, C., Zhu, F., Liu, X., Zhang, J., Wang, B., Xiang, H., Cheng, Z., Xiong, Y., Zhao, Y., Li, Y., Wang, X., \& Peng, Z. (2020). Clinical Characteristics of 138 Hospitalized Patients With 2019 Novel Coronavirus-Infected Pneumonia in Wuhan, China. JAMA, 323(11), 1061-1069. https://doi.org/10.1001/jama.2020.1585

2. Carter, C., Osborn, M., Agagah, G., Aedy, H., \& Notter, J. (2020). COVID-19 disease: invasive ventilation. Clinics in Integrated Care, 1, 100004. https://doi.org/10.1016/j.intcar.2020.100004

3. Yuki, K., Fujiogi, M., \& Koutsogiannaki, S. (2020). COVID-19 pathophysiology: A review. Clinical immunology (Orlando, Fla.), 215, 108427. https://doi.org/10.1016/j.clim.2020.108427

4. McNicholas, B., Cosgrave, D., Giacomini, C., Brennan, A., \& Laffey, J. G. (2020). Prone positioning in COVID-19 acute respiratory failure: just do it?. British journal of anaesthesia, 125(4),

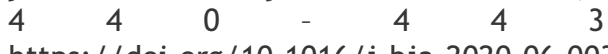
https://doi.org/10.1016/j.bja.2020.06.003

5. Martínez Ó, Nin N, Esteban A. Evidencias de la posición en decúbito prono para el tratamiento del síndrome de distrés respiratorio agudo: una puesta al día. Arch Bronconeumol. 1 de junio de 2009;45(6):291-6.

6. Sztajnbok J, Maselli-Schoueri JH, Cunha de Resende Brasil LM, et al. Prone positioning to improve oxygenation and relieve respiratory symptoms in awake, spontaneously breathing nonintubated patients with COVID-19 pneumonia. Respiratory Medicine Case Reports. 2020 ;30:101096. DOI: 10.1016/j.rmcr.2020.101096.

7. Mason R. J. (2020). Pathogenesis of COVID-19 from a cell biology perspective. The European respiratory journal, 55(4), 2000607. https: / / doi.org/10.1183/13993003.00607-2020

8. Dondorp, A. M., Hayat, M., Aryal, D., Beane, A., \& Schultz, M. J. (2020). Respiratory Support in COVID-19 Patients, with a Focus on ResourceLimited Settings. The American journal of tropical medicine and hygiene, 102(6), 1191-1197. https: / /doi.org/10.4269/ajtmh.20-0283

9. Guo, Y. R., Cao, Q. D., Hong, Z. S., Tan, Y. Y., Chen, S. D., Jin, H. J., Tan, K. S., Wang, D. Y., \& Yan, Y. (2020). The origin, transmission and clinical therapies on coronavirus disease 2019 (COVID-19) outbreak - an update on the status. Military Me d ical Re se a r ch, 7 (1), 11 . https: / / doi.org/10.1186/s40779-020-00240-0

10. Li, X., \& Ma, X. (2020). Acute respiratory failure in COVID-19: is it "typical" ARDS?. Critical care (London, England), 24(1), 198 . https://doi.org/10.1186/s13054-020-02911-9

11. Ksiazek, T. G., Erdman, D., Goldsmith, C. S., Zaki, S. R., Peret, T., Emery, S., Tong, S., Urbani, C., Comer, J. A., Lim, W., Rollin, P. E., Dowell, S. F., 
Ling, A. E., Humphrey, C. D., Shieh, W. J., Guarner, J., Paddock, C. D., Rota, P., Fields, B., DeRisi, J., ... SARS Working Group (2003). A novel coronavirus associated with severe acute respiratory syndrome. The New England journal of medicine, $348(20), 1953-1966$. https://doi.org/10.1056/NEJMoa030781

12. Zhou, F., Yu, T., Du, R., Fan, G., Liu, Y., Liu, Z., Xiang, J., Wang, Y., Song, B., Gu, X., Guan, L., Wei, Y., Li, H., Wu, X., Xu, J., Tu, S., Zhang, Y., Chen, H. , \& Cao, B. (2020). Clinical course and risk factors for mortality of adult inpatients with COVID-19 in Wuhan, China: a retrospective cohort study. Lancet (London, England), 395(10229), 1054-1062. https://doi.org/10.1016/S01406736(20)30566-3

13. Ng, Z., Tay, W. C., \& Ho, C. (2020). Awake prone positioning for non-intubated oxygen dependent COVID-19 pneumonia patients. The European respiratory journal, 56(1), 2001198. https: //doi.org/10.1183/13993003.01198-2020

14. Coppo A, Bellani G, Winterton D, Pierro MD, Soria $A$, Faverio $P$, et al. Feasibility and physiological effects of prone positioning in non-intubated patients with acute respiratory failure due to COVID-19 (PRON-COVID): a prospective cohort study. The Lancet Respiratory Medicine [Internet]. 19 de junio de 2020 [citado 4 de julio de

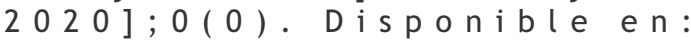
https://www.thelancet.com/journals/lanres/art icle/PIIS2213-2600(20)30268-X/abstract

15. Rialp Cervera G. Efectos del decúbito prono en el síndrome de distrés respiratorio agudo (SDRA). Med Intensiva. 1 de agosto de 2003;27(7):481-7.

16. Ghelichkhani, P., \& Esmaeili, M. (2020). Prone Position in Management of COVID-19 Patients; a Commentary. Archives of academic emergency medicine, 8(1), e48.

17. Beitler, J. R., Shaefi, S., Montesi, S. B., Devlin, A., Loring, S. H., Talmor, D., \& Malhotra, A. (2014). Prone positioning reduces mortality from acute respiratory distress syndrome in the low tidal volume era: a meta-analysis. Intensive care $\mathrm{m}$ e d i c i n e, 40 ( 3 ), $332-341$. https://doi.org/10.1007/s00134-013-3194-3

18. Chad, T., \& Sampson, C. (2020). Prone positioning in conscious patients on medical wards: A review of the evidence and its relevance to patients with COVID-19 infection. Clinical medicine (London, Eng $($ a nd), 20 (4), e 97 - e 103 . https://doi.org/10.7861/clinmed.2020-0179

19. Accoce, M., Plotnikow, G., Setten, M., Villalba, D., \& Galindez, P. (2017). DECUBITO PRONO: REVISIÓN NARRATIVA. Revista Argentina De Terapia Intensiva, 34(1), 63-75. Recuperado a partir de //revista.sati.org.ar/index.php/Ml/article/view 1458

20. Kallet R. H. (2015). A Comprehensive Review of Prone Position in ARDS. Respiratory care, 60(11), $\begin{array}{lllllllll}1 & 6 & 6 & 0 & - & 1 & 6 & 8 & 7\end{array}$

https://doi.org/10.4187/respcare.04271

21. Gattinoni, L., Busana, M., Giosa, L., Macrì, M. M., \& Quintel, M. (2019). Prone Positioning in Acute Respiratory Distress Syndrome. Seminars in respiratory and critical care medicine, 40(1), 94-100. https: / / doi.org/10.1055/s-0039-1685180

22. Lindahl S. (2020). Using the prone position could help to combat the development of fast hypoxia in some patients with COVID-19. Acta paediatrica (Oslo, Norway : 1992), 109(8), 1539-1544. https://doi.org/10.1111/apa.15382

23. Mezidi, M., \& Guérin, C. (2018). Effects of patient positioning on respiratory mechanics in mechanically ventilated ICU patients. Annals of translational medicine, 6(19), 384. https://doi.org/10.21037/atm.2018.05.50

24. Jiang, L. G., LeBaron, J., Bodnar, D., Caputo, N. D., Chang, B. P., Chiricolo, G., Flores, S., Kenny, J., Melville, L., Sayan, O. R., Sharma, M., Shemesh, A., Suh, E., \& Farmer, B. (2020). Conscious Proning: An Introduction of a Proning Protocol for Nonintubated, Awake, Hypoxic Emergency Department COVID-19 Patients. Academic emergency medicine : official journal of the Society for Academic Emergency Medicine, $27(7), \quad 566-569$. https://doi.org/10.1111/acem.14035

25. Ng, Z., Tay, W. C., \& Ho, C. (2020). Awake prone positioning for non-intubated oxygen dependent COVID-19 pneumonia patients. The European respiratory journal, 56(1), 2001198. https://doi.org/10.1183/13993003.01198-2020

26. Scaravilli, V., Grasselli, G., Castagna, L., Zanella, A., Isgrò, S., Lucchini, A., Patroniti, N., Bellani, G., \& Pesenti, A. (2015). Prone positioning improves oxygenation in spontaneously breathing nonintubated patients with hypoxemic acute respiratory failure: A retrospective study. Journal of critical care, 30(6), 1390-1394. https://doi.org/10.1016/j.jcrc.2015.07.008

27. Xu, Q., Wang, T., Qin, X., Jie, Y., Zha, L., \& Lu, W. (2020). Early awake prone position combined with high-flow nasal oxygen therapy in severe COVID19: a case series. Critical care (London, England), 24(1), 250. https://doi.org/10.1186/s13054-02002991-7

28. Elharrar, X., Trigui, Y., Dols, A. M., Touchon, F., Martinez, S., Prud'homme, E., \& Papazian, L. (2020). Use of Prone Positioning in Nonintubated Patients With COVID-19 and Hypoxemic Acute Respiratory Failure. JAMA, 323(22), 2336-2338. https://doi.org/10.1001/jama.2020.8255

29. Damarla, M., Zaeh, S., Niedermeyer, S., Merck, S., Niranjan-Azadi, A., Broderick, B., \& Punjabi, N. (2020). Prone positioning of nonintubated patients with COVID-19. American journal of respiratory and critical care medicine, 202(4), 604-606. https://doi.org/10.1164/rccm.202004- 
1331LE

30. Jochmans, S., Mazerand, S., Chelly, J., Pourcine, F., Sy, O., Thieulot-Rolin, N., Ellrodt, O., Mercier Des Rochettes, E., Michaud, G., SerbourceGoguel, J., Vinsonneau, C., Vong, L., \& Monchi, M. (2020). Duration of prone position sessions: a prospective cohort study. Annals of intensive care, 10(1), 66. https://doi.org/10.1186/s13613-02000683-7

31. Langer, M., Mascheroni, D., Marcolin, R., \& Gattinoni, L. (1988). The prone position in ARDS patients. A clinical study. Chest, 94(1), 103-107. https://doi.org/10.1378/chest.94.1.103

32. Papazian, L., Paladini, M. H., Bregeon, F., Huiart, L., Thirion, X., Saux, P., Jammes, Y., \& Auffray, J. P. (2001). Is a short trial of prone positioning sufficient to predict the improvement in oxygenation in patients with acute respiratory distress syndrome?. Intensive care medicine, 27 ( 6 ), $1044-1049$. https: / /doi.org/10.1007/s001340000799

33. Pappert, D., Rossaint, R., Slama, K., Grüning, T., \& Falke, K. J. (1994). Influence of positioning on ventilation-perfusion relationships in severe adult respiratory distress syndrome. Chest, 106(5), $\begin{array}{lllllllll}1 & 5 & 1 & 1 & - & 1 & 5 & 1 & 6\end{array}$. https://doi.org/10.1378/chest.106.5.1511

34. Romero, C. M., Cornejo, R. A., Gálvez, L. R., Llanos, O. P., Tobar, E. A., Berasaín, M. A., Arellano, D. H., Larrondo, J. F., \& Castro, J. S.
(2009). Extended prone position ventilation in severe acute respiratory distress syndrome: a pilot feasibility study. Journal of critical care, 24 ( 1 ), https: //doi.org/10.1016/j.jcrc.2008.02.005

35. Longhini, F., Bruni, A., Garofalo, E., Navalesi, P., Grasselli, G., Cosentini, R., Foti, G., Mattei, A., Ippolito, M. , Accurso, G., Vitale, F., Cortegiani, A., \& Gregoretti, C. (2020). Helmet continuous positive airway pressure and prone positioning: A proposal for an early management of COVID-19 patients. Pulmonology, 26(4), 186-191. https://doi.org/10.1016/j.pulmoe.2020.04.014

36. Armenta JMA, Terán ML, Cervantes VH.(2014). Ventilación pulmonar en decúbito prono y SDRA grave en Urgencias. Reporte de casos. Archivos de Medicina de Urgencia de México.26(6),30-37.

37. Guérin, Claude Prone position, Current Opinion in Critical Care: February 2014 - Volume 20 - Issue 1 p 92-97 doi: 10.1097/MCC.0000000000000059.

\section{Correspondencia}

Claudia Lizeth Uriol-Mantilla

Correo: clauumantila@gmail.com

\section{Revisión de pares}

Recibido: 15/10/2020

Aceptado: 15/12/2020 